\title{
4.3 CASSIOPEIA A - THE YOUNGEST KNOWN GALACTIC SUPERNOVA REMNANT
}

\author{
SIDNEY VAN DEN BERGH \\ David Dunlap Observatory, University of Toronto, Richmond Hill, Ontario, Canada
}

\begin{abstract}
Proper motion observations show that the explosion of the Cas A supernova took place in $\mathrm{AD} 1667 \pm 8(\mathrm{me})$. Individual moving knots have lifetimes $\sim 10 \mathrm{yr}$. In these fast-moving knots oxygen and argon are overabundant by a factor of at least seventy with respect to hydrogen and nitrogen. Nitrogen is found to be overabundant in quasi-stationary flocculi. This suggests that these flocculi were formed from material that was ejected from the outer layers of the pre-supernova before its explosion in AD 1667.
\end{abstract}

Following up the first accurate position determination of Cas A by Graham Smith in 1951, Baade and Minkowski (1954) were able to identify this radio source with a remarkable new type of emission nebula. These early optical observations established that the remnant of Cas A consists of two entirely distinct components: (1) A rapidly expanding system of knots and filaments that radiate strongly in [O $\mathrm{OII}$ ] and in [S $\mathrm{SI}$ ] and (2) A number of quasi-stationary flocculi that emit [NII] and $\mathrm{H} \alpha$.

Well-exposed plates of Cas A obtained in better than average seeing with the 200-in. telescope show approximately 130 nebulous knots. Of these about 100 are moving knots and approximately 30 are quasi-stationary flocculi. Intercomparison of plates taken some years apart (see Figure 1) shows that new knots are continually forming while old ones fade away. Available data on the decay of moving knots are adequately represented by the relation

$$
n(t)=n(0) e^{-t / \tau}
$$

with $n(0) \simeq 100$ and $\tau \simeq 10 \mathrm{y}$. The main uncertainty in this result is due to variations in plate quality caused by differences in seeing. The survival lifetime of large filaments, such as No. 1 of Baade and Minkowski (1954), is undoubtedly much longer than that of the average knot. Intercomparison of plates obtained in very good seeing suggests that the decay timescale of the smallest structural details in the remnant of Cas A is probably considerably shorter than ten years. Such fast changes indicate that the recombination timescale $\tau \simeq\left(\alpha_{\mathrm{rec}} \eta_{\mathrm{e}}\right)^{-1}$ is short and hence that the density in moving knots is high. This conclusion is supported by observations of the intensity ratio of the [SII] lines $\lambda \lambda 6716,6731$ which yields $\eta_{e} \simeq 1 \times 10^{4} \mathrm{~cm}^{-3}$.

New moving knots appear predominantly in regions in which other moving knots and filaments are already present. As a result no significant changes in the large-scale distribution of luminous nebulosity has occurred in Cas A during the last nineteen years. This observation suggests that luminous moving knots are fragments of the former supernova that are ploughing through stationary interstellar cloudbanks at hypersonic velocities. 


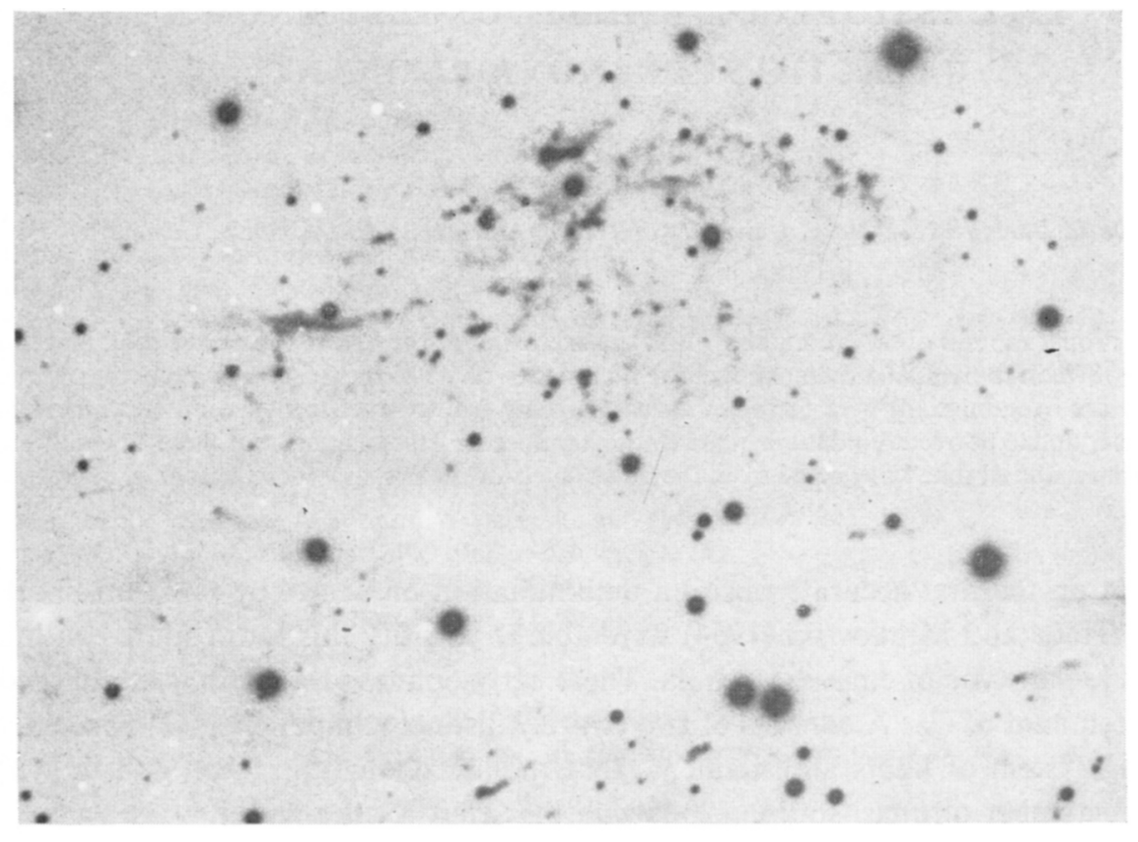

$[S \quad I]+\mathrm{H} \alpha$

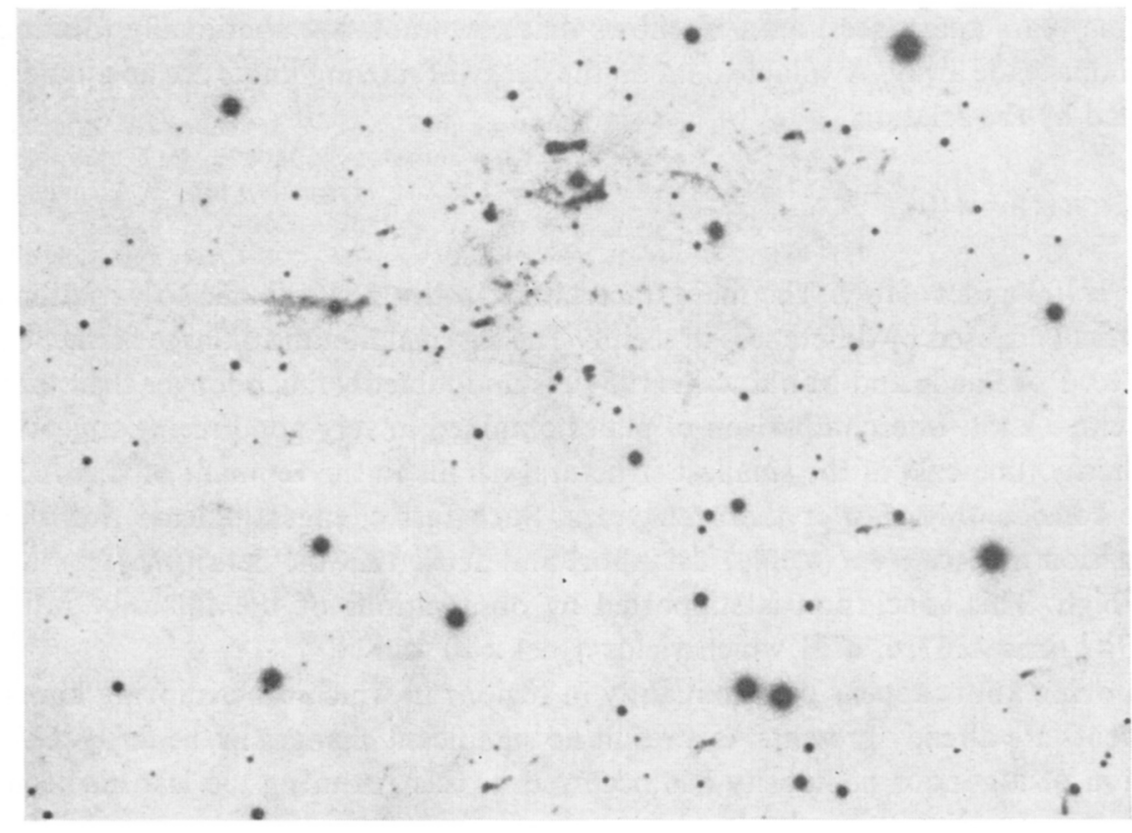

Fig. 1. Intercomparison of these two plates, which were both taken in red light, shows the general expansion of the optical remnant of Cas A. Note the rapid changes that have taken place in the small-scale structural details of the moving knots. 
If this conclusion is correct then the moving knots in Cas A should have a composition similar to that of the supernova from which they were formed. Furthermore the high densities that are observed in bright moving knots and the lack of observable deceleration (van den Bergh and Dodd, 1970) suggest that the material in moving knots cannot have been significantly contaminated by swept-up interstellar material.

Peimbert and van den Bergh (1971) have used observations of line intensity ratios to determine the chemical composition in moving knots and to estimate the absorption in the direction of Cas $\mathrm{A}$. The absorption is found to lie in the range $5<A_{V}<7$ mag. In moving knots oxygen is overabundant with respect to nitrogen by a factor of at least seventy. The sulphur-to-oxygen ratio is about two times lower in Cas A than it is in the Orion Nebula. Furthermore oxygen and argon are found to be overabundant by a factor of at least seventy with respect to hydrogen.

Observations of stationary flocculi show that nitrogen is overabundant with respect to hydrogen and oxygen. This suggests that stationary flocculi were formed when a preexisting circumstellar envelope was engulfed by the expanding supernova shell.

The bright moving nebulosity in Cas A is strongly concentrated in knots. In this respect Cas A differs from the Crab Nebula in which the moving nebulosity is mainly

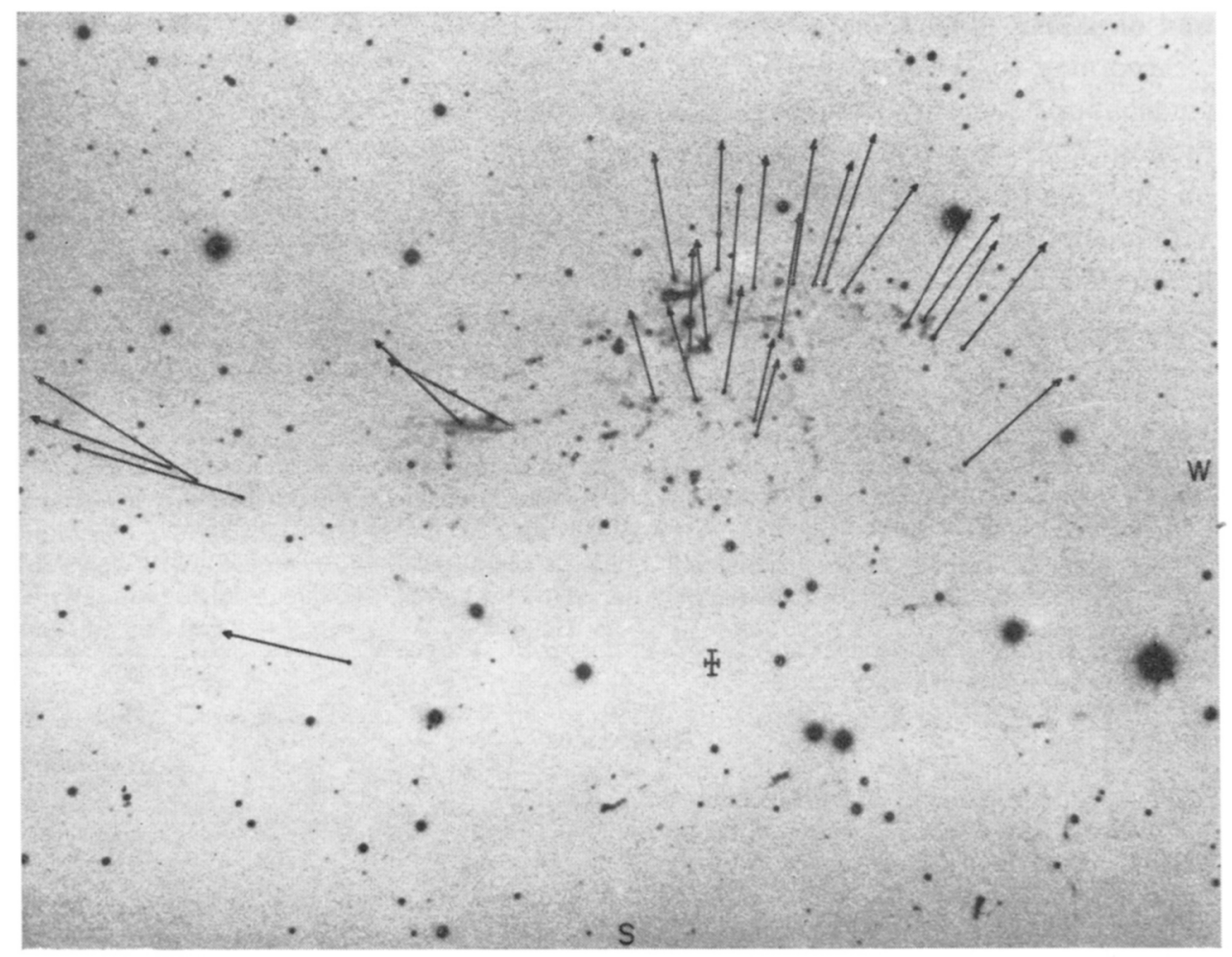

Fig. 2. The figure shows $100 \mathrm{y}$ proper motion vectors of individual knots of nebulosity. The centre of expansion of the optical remnant of Cas $\mathbf{A}$ is marked by a cross.

(Courtesy The Astrophysical Journal) 
concentrated in filaments. This may indicate that magnetic fields play a larger role in the expanding shell of the Crab than they do in Cas A. Radial velocity observations confirm that the nebulosity in Cas A consists of discrete knots with differing velocities. Velocity differences of up to $3000 \mathrm{~km} \mathrm{sec}^{-1}$ are observed for knots within a single filament. The origin of these large velocity differences remains a mystery. In most knots the half-width of the doppler profile of emission lines is about $200 \mathrm{~km} \mathrm{sec}^{-1}$. On direct plates typical moving knots are seen to have diameters of $2^{\prime \prime}$ corresponding to linear dimensions of about $1 \times 10^{+17} \mathrm{~cm}$.

Van den Bergh and Dodd (1970) have used sixteen 200-inch plates taken between 1951 and 1968 to study the expansion of the optical remnant of Cas A. Proper motions of individual knots are plotted in Figure 2. Assuming no deceleration the explosion of the supernova took place in $\mathrm{AD} 1667 \pm 8$ (me). In Table $\mathrm{I}$ the position of the centre of expansion that was obtained by van den Bergh and Dodd is compared with other determinations of the centre of Cas A. No star brighter than $V=22.5$ is visible within eight standard deviations of the observed centre of expansion. For $A_{V} \leqslant 7$ mag this result implies that any stellar remnant of Cas A must be intrinsically fainter than the pulsar in the Crab Nebula. In making such a comparison one should, of course, remember that the apparent brightness of a pulsar depends on the inclination of its axis of rotation.

According to Woltjer (1957) $V \simeq 19$ mag $(\operatorname{arcsec})^{-2}$ for the brightest part of the amorphous nebulosity that surrounds the Crab pulsar. With $A_{V} \simeq 7$ mag the corresponding surface brightness in Cas A would be well below the level of detectability on currently available plates.

Photoelectric photometry of stars in the direction of Cas A is reported in van den Bergh (1971a). A detailed description of the spectra of moving knots and stationary flocculi in Cas A is given in van den Bergh (1971b).

TABLE I

Centre of expansion of Cassiopeia A

\begin{tabular}{llll}
\hline & Period & $\alpha(1950)$ & $\delta(1950)$ \\
\hline van den Bergh and Dodd (1970) & $1951-1968$ & $23^{\mathrm{h}} 21^{\mathrm{m}} 11^{\mathrm{s}} .4 \pm 0^{\mathrm{s}} .2$ & $+58^{\circ} 32^{\prime} 18^{\prime \prime} .9 \pm 3^{\prime \prime} .1$ \\
Minkowski (1959) & $1951-1954$ & $2321 \quad 11.8 \pm 0.4$ & $+583216 \quad \pm 8$ \\
Rosenberg* (1970) & 1968 & $2321 \quad 9.5$ & +583222 \\
\hline
\end{tabular}

* Geometrical centre of radio source.

\section{References}

Baade, W. and Minkowski, R.: 1954, Astrophys. J. 119, 206.

Bergh, S. van den: 1971a, Astrophys. J. (in press).

Bergh, S. van den: 1971b, Astrophys. J. (in press).

Bergh, S. van den and Dodd, W. W.: 1970, Astrophys. J. 162, 485.

Minkowski, R.: 1959, IAU Symp. 9, p. 315.

Minkowski, R.: 1970, private communication.

Peimbert, M. and Bergh, S. van den: 1971 (in preparation).

Rosenberg, I.: 1970, Monthly Notices Roy. Astron. Soc. 147, 215.

Woltjer, L.: 1957, Bull. Astron. Inst. Neth. 13, 293. 


\section{Discussion}

R. C. Jennison: Several beautiful syntheses of Cassiopeia A have been performed in the range $1 \mathrm{GHz}$ to $3 \mathrm{GHz}$ in recent years but no structure has been mapped at lower frequencies since the original work by Jennison and Latham c. 1955. This low frequency $(127 \mathrm{MHz})$ phase sensitive synthesis showed a spur which was shortly afterwards observed photographically by Minkowski as a flare in position angle $70^{\circ}$. Many years later it appeared on the Cambridge high frequency syntheses, not as a spur, but as a break in the shell wall at the same position angle. I have suggested (Nature 207 (1965) 740) that the spur has a spectrum strongly favouring low frequency radio emission. I note that the Crab has a break in the shell and a faint short spur at optical frequencies would suggest that it may also have a low frequency enhancement in this direction. We certainly cannot ignore the flares in these supernovae and I shall be glad if Dr. van den Bergh can comment on the optical flare in Cassiopeia A.

S. van den Bergh: It would certainly be quite important to repeat these old observations. Recent Cambridge observations at short wavelengths do not show radio radiation at the position angle of the optical jet. If the jet should show up at long wavelengths it would, of course have to have quite a steep radio spectrum. Comparison of the Cambridge one-mile telescope map of Cas A with optical observations does not show a close correlation between optical and radio 'hotspots'.

$J$. E. Felten: You contemplated values of the visual extinction ranging from three to seven in magnitude. Is there any way of getting a better idea what the visual extinction is in this direction?

$S$. van den Bergh: You could measure the relative intensities of the far infrared sulphur lines and the ultraviolet sulphur lines which originate in the same upper level and so you know the theoretical (unobscured) intensity ratio. These observations are difficult and have not been made.

A.T. Moffet: Is there any way to tell whether there might be large differences in the obscuration in front of different parts of Cas A? The optical filaments are all seen on the northern edge, while the radio source is quite symmetrical.

$S$. van den Bergh: No. Intercomparison of 200-in. plates taken in the ultraviolet and in the infrared (where the absorption is relatively small) show the same large-scale structure of the expanding system of knots. Another argument against a large difference in absorption in the northern and in the southern parts of the remnant is that the stationary $\mathrm{H} \alpha$ flocculi can be seen both to the north and to the south of the centre of the expansion. I might add that some moving knots have also developed in the southern part of Cas A recently.

L. H. Aller: Some years ago Minkowski found that the Cassiopeia A filaments exhibited a spectrum with strong [OIII] lines but weak (or absent) $\mathrm{H}$. We suggested that perhaps the filament was excited by collisions with the interstellar medium such that the ambient electron temperature was not high enough to collisionally excite $\mathrm{H}$. The absence of $\mathrm{H}$ lines is difficult to understand. If doubly ionized oxygen is present, why do recombination lines of hydrogen not appear? Perhaps the ionization of oxygen is by radiation and the hydrogen recombination lines are too weak. Possibly the supernova material is deficient in $\mathrm{H}$ and what we observe is excited supernova material. But then why is the interstellar gas not excited as it is 'bulldozed' by the supernova shell? Admittedly the density of the bulldozed interstellar matter is low but we should see some $\mathrm{H}$ contribution. A very severe difficulty for theoreticians is imposed by the disappearance and re-appearance of filaments. Certainly the simple interpretation which Minkowski and I gave must now be discarded and strong timedependent effects must be included. 